\title{
Galaxy bulges at mid- and high-redshift
}

\author{
Christopher J. Conselice ${ }^{1}$ \\ ${ }^{1}$ School of Physics and Astronomy, University of Nottingham, Nottingham, U.K.
}

\begin{abstract}
Bulges are a major galaxy component in the nearby universe, and are one of the primary features that differentiates and defines galaxies. The origin of bulges can be directly probed in part by examining distant galaxies to search for high redshift bulges, and to study the properties of bulges in formation. We review the evidence for bulges at high redshift in this article, and how by studying bulges through a variety of approaches, including through morphological, colour, and stellar mass selection, we can determine when and how these systems assembled. We argue that the majority of the most massive 'classical' bulges are in place by $z \sim 1.5-2$, and likely formed very early through major mergers. Other, likely lower mass, bulges form through a secular process along with their disks. Direct observations suggest that these two formation processes are occurring, as spheroids are commonly seen at $z>1$, as are disks and spiral galaxies in the form of luminous diffuse objects, clump-clusters, and chain galaxies. However, bulge+disk systems are relatively rare until $z \sim 1$, suggesting that this structural assembly occurred relatively late.
\end{abstract}

\section{Introduction}

Galaxy bulges are one of the major components of nearby galaxies, and help define the Hubble classification sequence. Bulges are very common, and are found in a large fraction $(>50 \%)$ of all nearby galaxies. Depending on how bulges are defined, a large fraction of the light, and stellar mass, in nearby galaxies are contained within these systems (e.g., Benson et al. 2002). Understanding the formation modes and history of bulges is therefore clearly important for our understanding of all galaxies, as well as for uncovering how and when the Hubble sequence assembled.

This paper reviews our current understanding of mid- and high-redshift galaxy bulges and/or the progenitors of modern galaxy bulges. Since galaxy bulges are fairly common in the local universe, we should be able to explore in a fairly straightforward manner the formation and evolution of these systems by looking at more distant galaxies. We describe in this review the current evidence for the existence of bulges at higher redshifts, based on observations of spheroids and spirals/disks, and how the physical evolution of these galaxies suggest how bulges are assembling.

Before we can study bulges in the early universe we must be clear about the definition of what makes a bulge, and how to locate their progenitors. This is a primary question to address, but one in which there is no generally agreed upon answer. In this review we examine the evolution of bulges using several techniques and methods. One method is to assume that a bulge must be attached to a disk galaxy, and that all large, massive disk galaxies have a bulge, or a proto-bulge, at their centre. The other working definition is to assume that a bulge is a spheroid that acquired a disk through an accretion process, or disk assembly. Studying bulge progenitors thus requires that we identify massive or spheroid galaxies at early times. We can then determine when the most massive bulges formed their stellar mass, if not already their structure/morphological state. We argue that many very massive bulges are formed by $z \sim 1.5-2$, based on the number densities of massive galaxies at early times. 
The other approach for finding bulges at high redshift is to search for disk galaxies in formation. This can be effectively done by searching for what appears through quantitative and qualitative methods to be disk galaxies at $z>1$. Features used to find these disk galaxies include: spiral arms, bars, star forming knots, and exponential or shallower light profiles. These proto-disks include the morphologically and luminosity defined: luminous diffuse objects (LDOs), clump-clusters, and chain galaxies. While normal appearing disk plus bulge systems are rare at $z>1$, there are many disk-like candidates, and bulge candidates, at higher redshifts whose properties can be studied. Observations show that these galaxies are undergoing a significant amount of star formation, and are often composed of multiple clumps of young stars that are potentially producing a bulge through secular evolution.

The current evidence at high-redshift suggests that more than one mechanism is responsible for the creation of bulge+disk systems. While spheroids and disk-like galaxies can be found at $z>1$, systems which would be identified as bulge+disks are relatively rare at these epochs, suggesting that the full Hubble sequence, and disk+bulge galaxy morphologies, are established relatively late. Throughout this paper we use the standard cosmology of $\mathrm{H}_{0}=70 \mathrm{~km} \mathrm{~s}^{-1} \mathrm{Mpc}^{-1}$, and $\Omega_{\mathrm{m}}=1-\Omega_{\Lambda}=0.3$, and a Chabrier IMF for stellar mass measurements, unless otherwise noted.

\section{Identifying High-Redshift Bulges}

\subsection{Nearby Bulges: A $z=0$ Benchmark}

Galaxies bulges are well studied in the nearby universe (e.g., Kormendy \& Kennicutt 2004), and observations of nearby disk+bulges continue to reveal important information on their formation (e.g., Lanyon-Foster et al. 2007; Drory \& Fisher 2007). Before we can effectively explore the formation and presence of mid- and high-redshift bulges it is important to quantify the number of these systems in the nearby universe to serve as a bench-mark for the maximum number of systems we expect to find at earlier times. This can be done in a number of ways, including investigating the number of bulges, or galaxies which host bulges, in the nearby universe.

Within the nearby universe, bulge-dominated galaxies are the most common galaxy type. Galaxies classified as early-type disks are found with a number density of $\sim 1.3 \pm$ $0.3 \times 10^{6} \mathrm{Gpc}^{-3} \mathrm{~h}_{100}^{3}$, with a similar number of late-type disks (Conselice 2006a), which also generally host bulges. Based on these densities we should easily find and detect bulges, or bulge progenitors, at higher redshifts. For example, between $0.2<z<1$ there should be 20 galaxies with massive bulges in the Hubble Deep Field, 1000 within the GOODS-fields, 2000 within the Extended Groth Strip, and 20,000 within the COSMOS field. A similar number of bulges/progenitors should be found at higher redshifts in these fields. Therefore, there is no lack of opportunity, even within relatively small Hubble Space Telescope imaging areas, to locate and study bulges at higher redshifts.

\subsection{High Redshift Bulges as Spheroids}

The dominate paradigm of galaxy formation is based on the successful $\Lambda$-dominated cold dark matter theory of structure formation. Within this picture, bulges, and spheroids in general, are the oldest galaxy components in the universe, and formed before the disk galaxies we see today. Within this formation scenario, which still lacks much observational basis, these spheroids and bulges are formed through the mergers of primordial galaxies which are rotationally supported (e.g., Baugh et al. 1998).

If galaxy bulges form in an inside-out fashion, such that the bulge is present before the disk, then looking for galaxy spheroids at mid- and high-redshift is an excellent way 

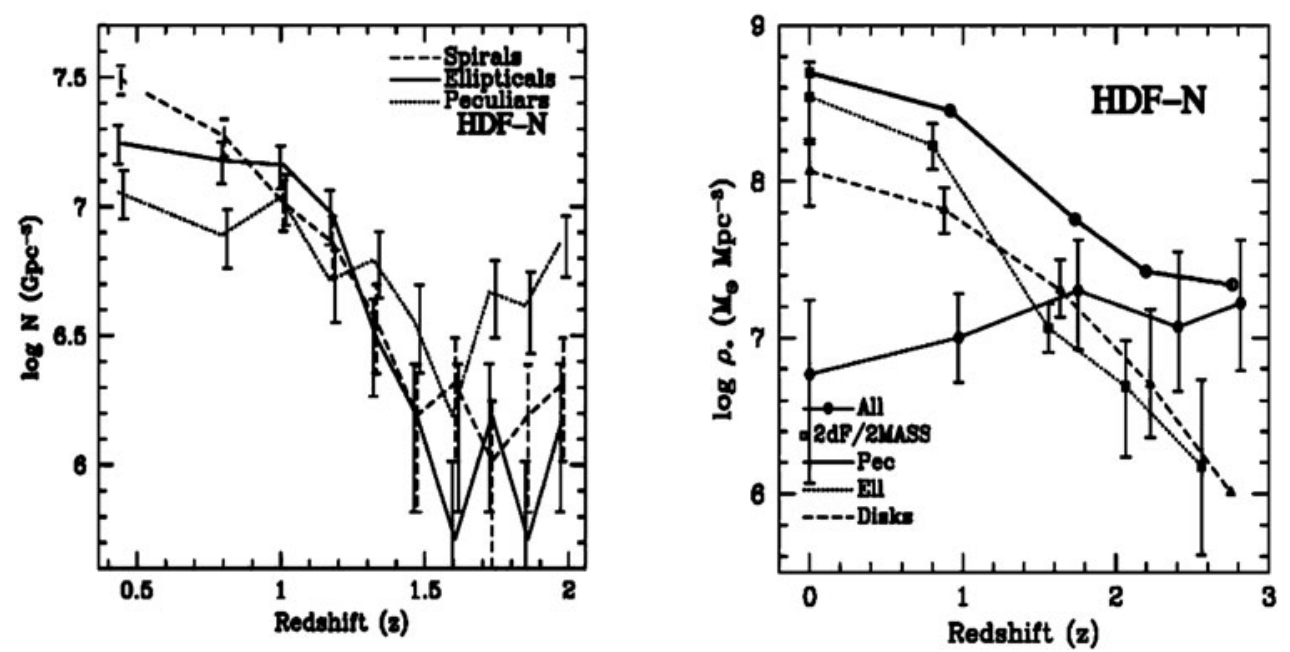

Figure 1. The relative contributions of various galaxy types to the galaxy population and the integrated stellar mass density as a function of redshift. The left panel shows the number density evolution for galaxies at $I<27$ up to $z \sim 3$. The right panel shows the evolution in the fraction of the galaxy stellar mass density associated with galaxies of various morphological types.

to study the progenitors of galaxy bulges, and how they form. There are several ways to study distant spheroids that may be the progenitors of bulges in modern disk galaxies. The most obvious examples are to search for spheroids morphologically, to search for red bright galaxies, and to locate massive galaxies at high redshift.

The first way to approach this problem is to determine how galaxy morphology evolves with cosmic time. Currently, the only field where this can be carried out, without having to account for morphological k-corrections, is within the Hubble Deep Field-North (HDFN) (Conselice et al. 2005a). Figure 1 shows the evolution of the relative contributions of spheroids, disks and peculiars to the galaxy population as a function of redshift. As can be seen at $z<1$, spheroids and disks are the dominate galaxy population, while peculiar galaxies are the most prominent at higher redshifts.

Based on this, we can conclude that while the stars that make up bulges might be present at high redshift $(z>1)$, these systems are not in their nearby relaxed morphological state, at least not in the same number that they are found at $z<1$. This implies that at least some evolution is occurring within bulges at $z>1.5$, and that very few, if any, disk+bulge systems are present at $z>1.5$. The final structural assembly of disks with bulges thus occurs relatively late in the history of the universe. While the HDF-N is a small field of view, other studies have found a similar lack of conclusive examples of bulges+disk systems at higher redshifts (e.g., Conselice et al. 2003a, 2005a; Papovich et al. 2005; Ravindranath et al. 2006; Conselice et al. 2007a).

While bulge+disk systems are not present in a high abundance at higher redshifts, it is possible and as we shall argue, likely, that bulge progenitors are present at $z>1$. If we examine galaxy bulges as massive galaxies, then we can measure how much of the stellar mass within massive galaxies has formed at relatively high redshifts. This was recently examined by Conselice et al. (2007b) who found that the majority of massive galaxies with $\mathrm{M}_{*}>10^{11} \mathrm{M}_{\odot}$ are present by $z \sim 1$, while those of very high mass, with $\mathrm{M}_{*}>$ $10^{11.5} \mathrm{M}_{\odot}$ are statistically present at their $z \sim 0$ number and mass densities by $z \sim 2$ (Figure 2) (see also e.g., Fontana et al. 2004; Daddi et al. 2004). 

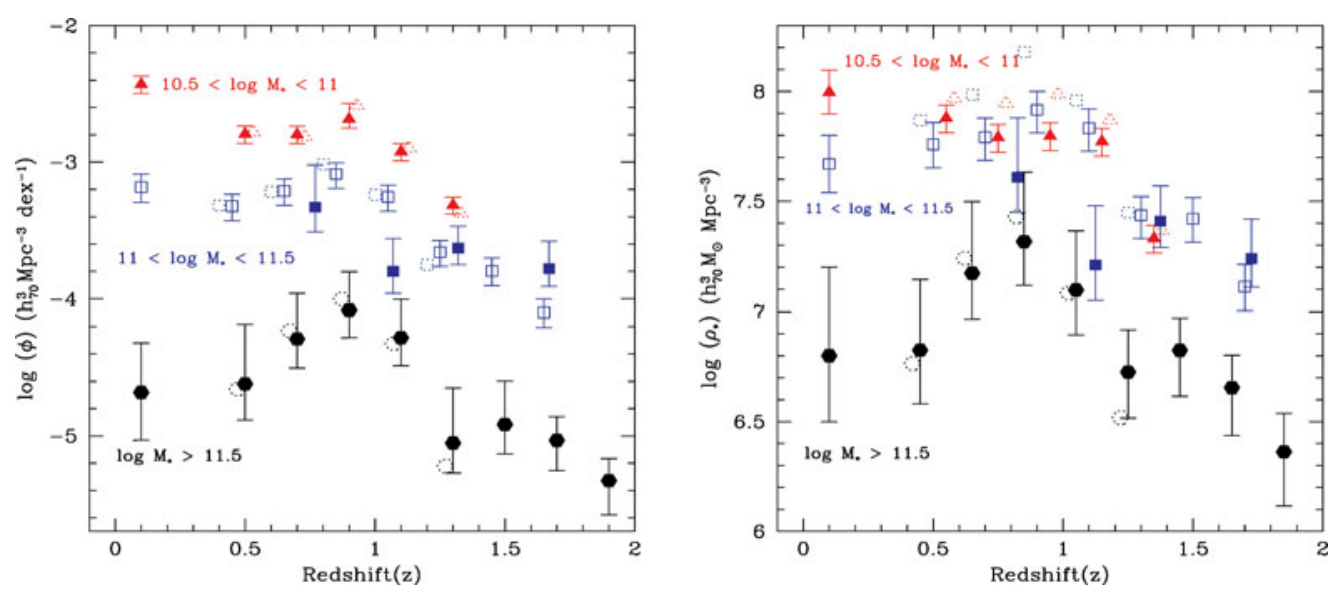

Figure 2. Left panel: the evolution in number densities for galaxies of various masses between $z \sim 0.4-1.4$. Right panel: the stellar mass density evolution as a function of galaxy mass at the same redshift intervals. The error bars listed on both the number and mass densities reflect uncertainties from stellar mass errors, as well as cosmic variance, and counting statistics.

We can use Figure 2 to argue that most of the stellar mass in the most massive bulges was likely in place by $z \sim 1.5-2$. The reason for this is that galaxies with masses $\mathrm{M}_{*}>$ $10^{11} \mathrm{M}_{\odot}$ are nearly all present by these redshifts, and in the local universe roughly $40 \%$ of all galaxies with $\mathrm{M}_{*}>10^{11} \mathrm{M}_{\odot}$ are disk galaxies (Conselice 2006a). This implies that a fairly large fraction of the massive galaxies we see at high redshift are the progenitors of bulge like galaxies. While most of these massive galaxies do not have disks surrounding them, we find that a fraction however are within spiral like galaxies (Conselice et al. 2007b).

\subsection{High Redshift Bulges as Disks}

Another way to search for high-redshift bulges is to find examples of bulges+disks, or pure disk galaxies, that might be forming bulges at $z>1$. This is an important exercise to carry out as it allows us to directly measure when, and perhaps how, the Hubble sequence was put into place. It also lets us determine whether bulge formation is an outside-in process, or an inside-out process, since if we found significant numbers of disks in formation that did not yet have bulges this would imply that the bulges form after their disks are in place.

The evidence for disk galaxies at high redshift is limited, and in some cases ambiguous. There are several reasons for this, including that disk galaxies are probably different morphologically in the past than they are today, making them difficult to identify. Also, the majority of high resolution Hubble imaging probes the rest-frame ultra-violet at $z>1.5$, making if difficult to identify disks+bulges (e.g., Windhorst et al. 2002; MagerTaylor et al. 2007). There are however many examples of disk galaxy candidates at high redshifts that are almost certain to be the progenitors of the disk galaxies we see today.

The first instance of large disk galaxies seen at high redshift originated from deep Hubble Space Telescope imaging in the Hubble Deep Field South (Labbe et al. 2003), and the GOODS fields (Conselice et al. 2004). These early studies found examples of galaxies that appear to have disk-like features, such spiral arms, bulges, bars, and clumpy star forming regions. Conselice et al. (2004) took this analysis further by investigating a population of possible proto-disk galaxies called luminous diffuse objects (LDOs) which are located by their low light concentrations, high luminosities, and large sizes (Figure 3). 

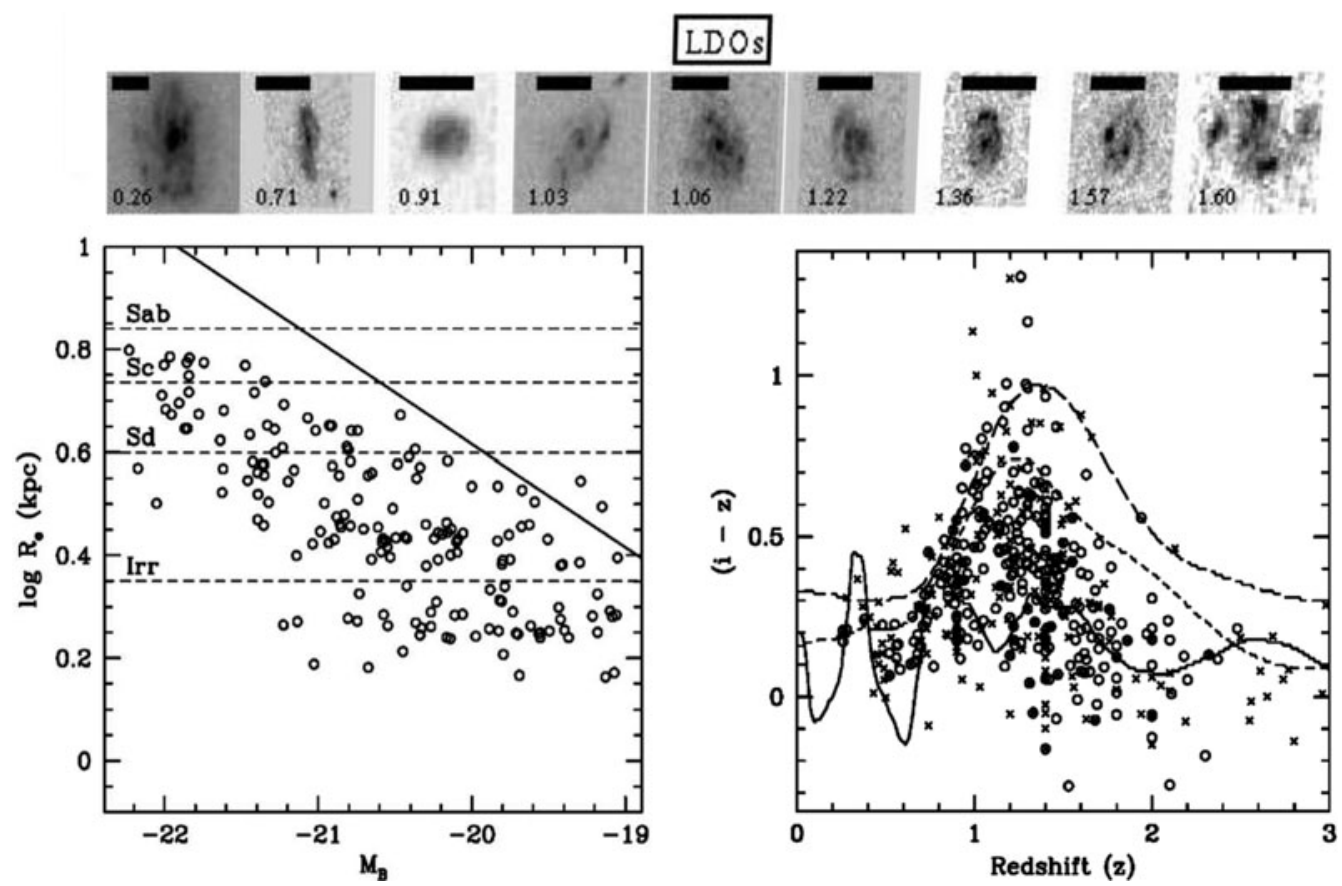

Figure 3. Top: images of luminous diffuse objects (LDOs) at $z<1.5$. Left: the size-absolute luminosity relation for the LDOs with the sizes of nearby galaxy types labelled and the relation for nearby disks shown as a solid line. Right: the observed $(i-z)$ colours for LDOs, showing a diversity of colour at different redshifts. The lines are, from bluest to reddest: starburst (solid line), Scd (dashed), and Sbc (long dashed).

These galaxies are found in abundance at $z>1$, with most systems at $1<z<2$. The size-luminosity relation for the LDOs is similar to nearby disk galaxies, but with a luminosity offset of a few magnitudes. The spectral energy distributions of these galaxies also suggests that they are undergoing starbursts (Figure 3).

Proto-disk galaxies have furthermore been studied in great detail by e.g., Elmegreen et al. (2007, and references therein). These studies find that a large fraction of all galaxies at $z>1$ are composed of clumpy light made up of very young $(\sim 100 \mathrm{~s}$ Myr $)$ star clusters, which have masses ranging from $10^{7}-10^{9} \mathrm{M}_{\odot}$. These clusters are found in both the classical chain galaxies, as well as in the clump-clusters. As shown by Elmegreen \& Elmegreen (2004) these two galaxy types are taken from the same population, with chain galaxies the edge-on version of clump-clusters. The evidence for this includes the fact that these two galaxy populations have similar sizes, magnitudes, and clump-cluster properties, as well as a combined axial ratio distribution expected for a disk population (Elmegreen \& Elmegreen 2005). Furthermore, more recent NICMOS observations of clump-clusters and LDOs suggest that their massive clusters are intrinsic to the galaxy, and are not lower mass star forming knots (Elmegreen et al. 2007).

\subsection{Kinematics of Disks at $z>0.5$}

Most of the above discussion relates to the morphological properties of distant galaxies. Another area where there is growing information concerning disk galaxies at mid- and high-redshifts is through the use of internal kinematics. For example, based on rotation 


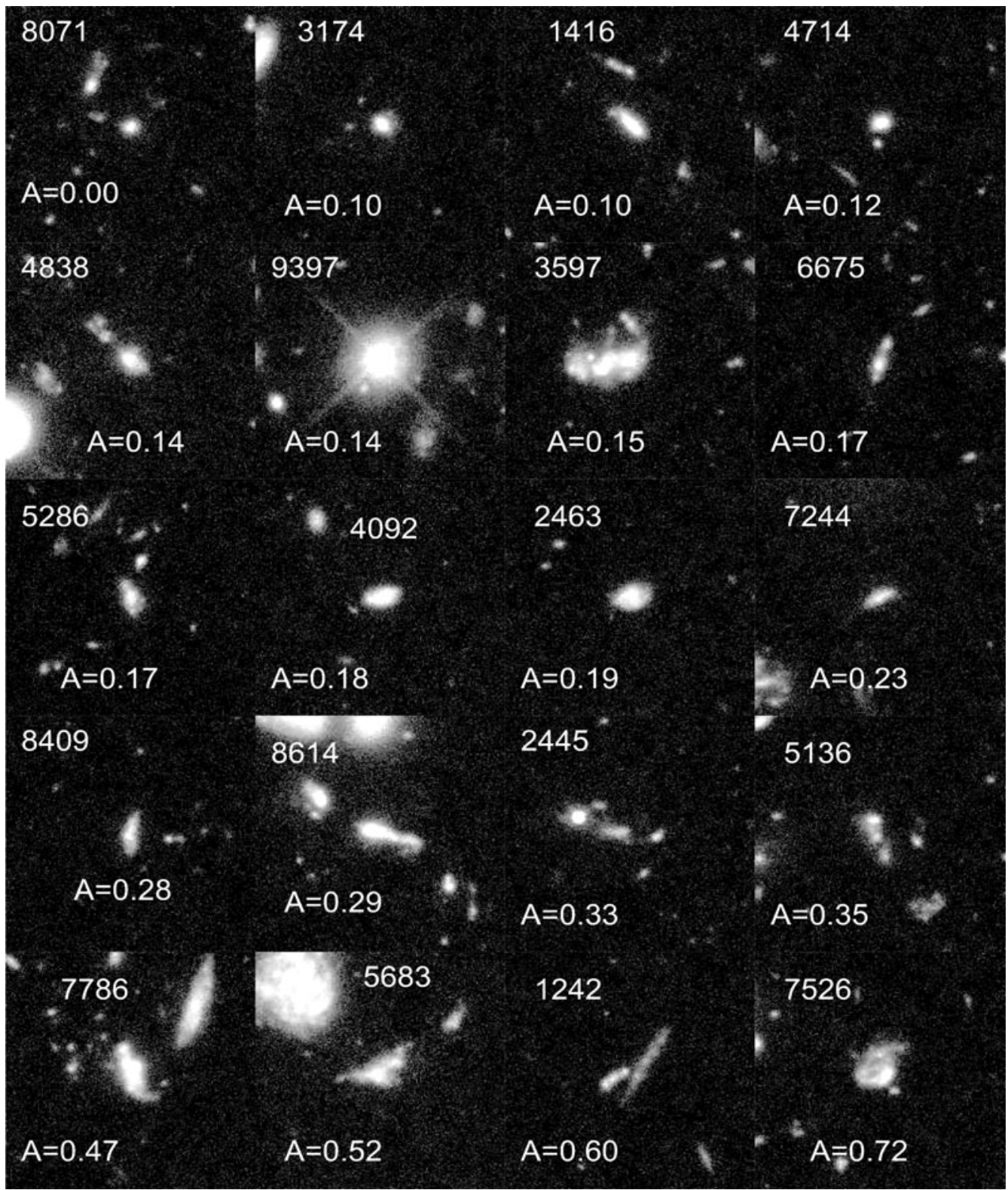

Figure 4. Images of high redshift galaxies as seen in the Hubble Ultra Deep Field. This example shows images of galaxies at $2.2<z<3.0$ and with stellar masses $\mathrm{M}_{*}>10^{10} \mathrm{M}_{\odot}$. The bottom number is the asymmetry for each system, and the upper number is its ID.

curves, it is possible to find morphologically selected disks with well ordered rotation up to $z \sim 1.2$, and to study the evolution of disk galaxy scaling relations (e.g., Vogt et al. 1997; Bohm et al. 2004; Ravindranath et al. 2004).

Detailed analyses of mid-redshift disk galaxies suggest that the formation of disks at $z<1.2$ is hierarchical. We know that disk galaxies at $z<1.2$ are undergoing star formation (e.g., Wolf et al. 2005), and based on their sizes, it appears that the largest disk galaxies already have their maximum size, and roughly $z \sim 0$ morphological properties 
by $z \sim 1$ (e.g., Jogee et al. 2004; Ravindranath et al. 2004). Thus, it might appear that the star formation within disks is occurring due to a conversion of existing gas into stars through the existing disks.

However, by comparing the stellar and total masses of disk galaxies at $0.2<z<1.2$, Conselice et al. (2005b) argue that disk galaxies grow hierarchically during this epoch. This can be seen by the fact that the ratio of disk galaxy stellar to total mass does not evolve at $z<1.2$. If the gas which star formation at $z<1.2$ arises is present within disks at $z \sim 1.2$, then the ratio of stellar to total mass would grow with time as gas is converted into stars. Since this ratio of masses is constant, it implies that there is gas and dark matter accretion onto disks during this time, and thus disk formation is largely hierarchical at $z<1.2$.

This observation is for disk galaxies that are mature morphologically at $z<1.2$, and does not address how disk galaxy formation occurs at earlier times. While we have discussed several examples of morphologically selected disk galaxies at higher redshifts, there are now several studies which have found evidence for rotating disks, and have studied morphologically peculiar galaxies at $z>1.5$ in the near-infrared with long-slit and integral field spectrographs to search for rotation, and measure internal kinematics.

Based on these observations, there are several examples of galaxies which appear to be rotating disks at these redshifts, with rotation rates $>200 \mathrm{~km} \mathrm{~s}^{-1}$. Many of these galaxies also show a large velocity dispersion, and some morphologically appearing 'disks' do not have any obvious rotation along their major axis (e.g., Erb et al. 2004), although the limited spatial extent of these observations makes it difficult to predict how a rotating disk would appear, especially if still in formation (e.g., Bournaud et al. 2007).

Integral field spectroscopy has however found that the majority of star forming selected galaxies at $z \sim 2$ have velocity gradients of up to $400 \mathrm{~km} \mathrm{~s}^{-1}$ (e.g., Forster Schreiber et al. 2004; Bouche et al. 2007). Some of these systems have morphologies and kinematics that suggest they are disks, while it is difficult to distinguish rotation from galaxy merging in other systems (Forster Schreiber et al. 2004). There are more certain examples of largescale rotating disks, using adaptive optics observations, such as the system at $z=2.38$ studied by Genzel et al. (2006), who find a disk galaxy with $\mathrm{V}_{\text {circ }}=230 \mathrm{~km} \mathrm{~s}^{-1}$, size 4.5 $\mathrm{kpc}$, and a total mass of $\sim 10^{11} \mathrm{M}_{\odot}$.

\section{Bulge Formation Mechanisms}

\subsection{Galaxy Merging}

While it is generally believed that bulges may have different formation mechanisms (e.g., Kormendy \& Kennicutt 2004), with the classical bulges forming through mergers, and the 'pseudo-bulges' forming through secular processes, this has not yet been directly shown or argued using high redshift data.

What we do know from $\S 2.2$ is that most of the stellar mass in the most massive galaxies is largely in place by $z \sim 1-2$, and it is likely that this epoch marks the end of the classical bulge formation phase, and we must probe higher redshifts to determine how these massive bulges form.

One of the ways to do this is to investigate the merger fraction, and merger rate, for massive galaxies, with $\mathrm{M}_{*}>10^{10} \mathrm{M}_{\odot}$ at $z>2$ (Conselice et al. 2003a; Conselice et al. 2007c, in prep; Figure 4 shows example Hubble images of these systems). Through the quantitative CAS structural parameters (e.g., Conselice et al. 2000a,b; Bershady et al. 2000; Conselice et al. 2002; Conselice 2003) we can identify major mergers at mid- and high-redshifts (e.g., Conselice et al. 2003a,b; Conselice 2006b; Pope et al. 2005; Bridge 

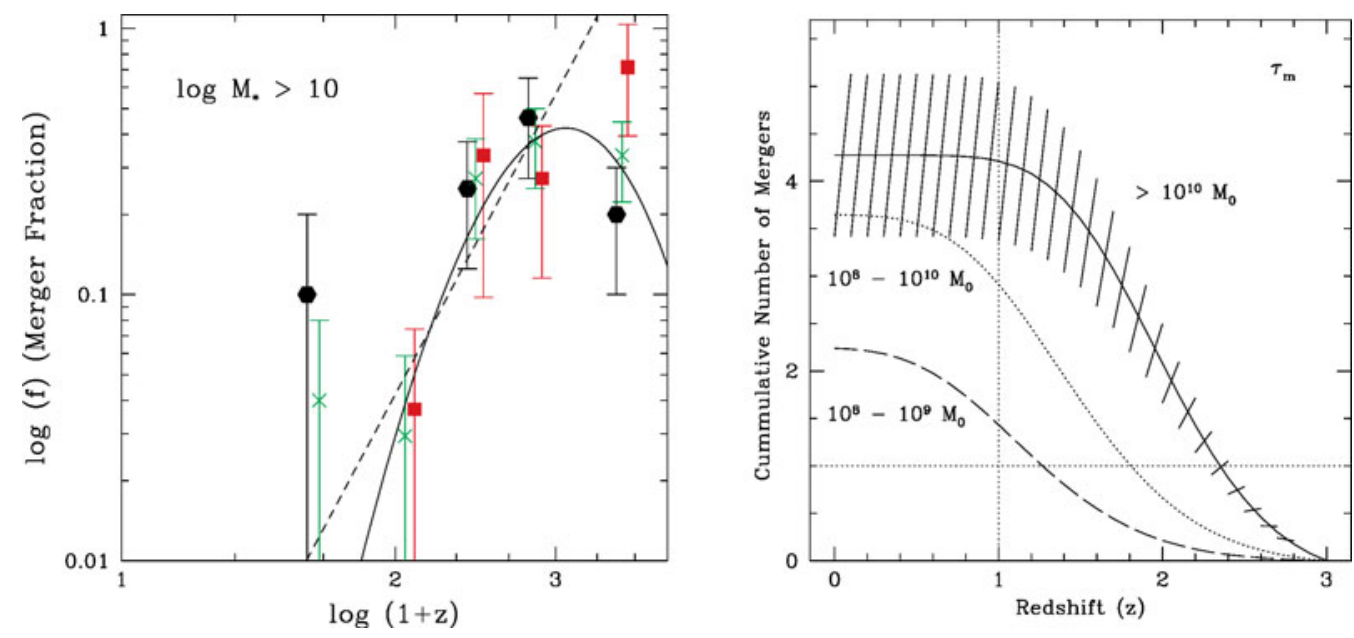

Figure 5. Left panel: The merger fraction for galaxies with stellar masses $M_{*}>10^{10} M_{\odot}$ up to $z \sim 3$ in the Hubble Ultra-Deep Field (red boxes) and the Hubble Deep Field-North (solid circles) and a combined sample (green crosses). Right panel: the evolution of the cumulative number of mergers occurring within galaxies at $z<3$ (Conselice et al. 2007, in prep).

et al. 2007). What these studies have found is that the most massive galaxies, for which we have rest-frame optical morphologies, appear peculiar at $z>2$ (Conselice et al. 2005a), and are likely in a major merger phase (e.g., Conselice et al. 2003a, 2007c).

By examining the evolution of the merger fraction with time, and by using derived time-scales for merging from N-body simulations (Conselice 2006b), we can determine the merger history for massive galaxies at $z<3$. The result of this, using a combined Hubble Ultra-Deep Field and Hubble Deep Field-North sample, is shown in Figure 5. These results show that the most massive galaxies with $\mathrm{M}_{*}>10^{10} \mathrm{M}_{\odot}$ at $z \sim 2.5$ undergo on average 4-5 major mergers at $z<3$, but that most of this merging occurs at $z>1.5$. This suggests that the most massive galaxies, and thus the most massive bulges, formed in major mergers at $z>2$.

\subsection{Disk Formation}

It is generally agreed that disks themselves cannot form through the merger process, at least in mergers that do not include significant amount of gas that is ejected and falls back onto a spheroid. In general, to form a disk, it is necessary to accrete gas from the intergalactic medium onto an existing bulge, or onto itself, to form a pure disk galaxy. This process appears likely to be the formation mechanism for at least some of the clump-clusters or LDOs which do not have any obvious bulges.

This formation process has been investigated by several teams who have simulated how a rotating disk made purely of gas will form stars (e.g., Noguchi 1999; Immeli et al. 2004; Bournaud et al. 2007). All of these studies have found that a gas rich rotating disk will fragment into large star clusters throughout, producing a morphology similar to the LDOs, clump-clusters, and chain galaxies. The evolution of these disks is driven largely by the interaction of these star clusters. In simulations these clusters lose angular momentum, dissolve, and disturb the underlying disk to such a degree that they are able to form a bulge in the centre of the disk, and later an exponential-like profile for the disk itself (Bournaud et al. 2007).

These simulations are also able to address the life-time of this clump-cluster phase within these systems. The latest simulations from Bournaud et al. (2007) suggest that 
this cluster phase lasts for roughly $0.5 \mathrm{Gyr}$, and only occurs once in the life-time of a disk. Before this, the galaxy is not forming stars, and after this phase, the galaxy appears as a disk+bulge system within the simulation.

Using high resolution Hubble imaging, we can determine directly how the properties of bulges and disks evolve at $z<1.2$, where both of these components are found in the same galaxy. An early study by Ellis et al. (2001) found that the bulges of disk galaxies at $z<1.2$ are bluer than pure spheroids at similar redshifts. More recently, Koo et al. (2005) found the opposite conclusion - that the bulges of spiral galaxies are red, and likely as old as spheroids at similar redshifts. Both studies however find that the disks of these distant systems are either bluer, or have similar colours, suggesting that bulges are older than their disks, or more likely that disks have had a more recent episode of star formation. However, not all 'spheroids' are old, as there are examples undergoing star formation at $z<1$ (e.g., Stanford et al. 2004; Teplitz et al. 2006).

\section{Conclusions and Future Outlook}

The study of bulges at mid- and high-redshifts is a very active field which will advance greatly during the next few years with high resolution near-infrared imaging with groundbased adaptive optics and with WFC3 on Hubble. We can however draw some conclusions about the way that bulges form based on current observations of the distant universe.

Observations show that there are many disk galaxies, as selected morphologically, up to $z \sim 1$, with a similar number density as disks today. However, at higher redshifts disk galaxies, and in particular disk galaxies with bulges, are rare. Most galaxies at $z>1.5$ appear peculiar in appearance. Therefore, to study bulges at $z>1$ we must search for bulge progenitors. If we consider bulges as massive spheroids, we find that most of these massive $\mathrm{M}_{*}>10^{11} \mathrm{M}_{\odot}$ galaxies are formed by $z \sim 1.5-2$. Since roughly $40 \%$ of $\mathrm{M}_{*}>$ $10^{11} \mathrm{M}_{\odot}$ galaxies at $z \sim 0$ are massive disk galaxies, this suggests that a large fraction of the stars in the most massive bulges are in place by this time.

However, finding bonafide disks forming around spheroids is difficult, and few convincing massive bulge+disks systems have been identified at $z>1.5$ (e.g., Figure 4). This is likely partially due to the lack of wide-area high-resolution near-infrared imaging of distant galaxies. There are however many examples of disk galaxies that appear to be forming through a secular process, including perhaps the establishment of a bulge. Examples of these are the clumpy galaxies at $z>2$ identified as luminous diffuse objects (LDOs), clump-clusters, and chain galaxies, that are likely a subset of the modern disk galaxy population in formation. Simulations show that the clumps in these galaxies interact over a few $100 \mathrm{Myr}$ to form a bulge (e.g., Bournaud et al. 2007).

In the future, imaging observations with adaptive optics and the WFC3 on HST and integral field spectroscopy in the NIR will greatly advance the study of galaxy bulges at mid- and high-redshifts. What is not clear yet is the relative role of the clump-cluster vs. gas accretion on massive spheroids to form bulge+disk systems. Identifying disks and bulges in formation through kinematics and high-resolution NIR imaging at $z>1$ will ultimately reveal how bulges are established in disks, providing complementary information obtain from nearby bulges.

\section{References}

Baugh, C. M., Cole, S., Frenk, C. S., \& Lacey, C. G. 1998, ApJ, 498, 504

Benson, A. J., Frenk, C. S., \& Sharples, R. M. 2002, ApJ, 574, 104

Bershady, M. A., Jangren, J. A., \& Conselice, C. J. 2000, AJ, 119, 2645 
Bohm, A., et al. 2004, A\&A, 420, 97

Bournaud, F., Elmegreen, B. G., \& Elmegreen, D. M. 2007, arXiv:0708.0306

Bridge, C., et al. 2007, ApJ, 659, 931

Conselice, C. J., Bershady, M. A., \& Jangren, A. 2000a, ApJ, 529, 886

Conselice, C. J., Bershady, M. A., \& Gallagher, J. S. 2000b, A\&A, 354, 21L

Conselice, C. J., Gallagher, J. S. III, \& Wyse, R. F. G. 2002, AJ, 123, 2246

Conselice, C. J. 2003, ApJS, 147, 1

Conselice, C. J., Bershady, M. A., Dickinson, M., \& Papovich, C. 2003a, AJ, 126, 1183

Conselice, C. J., Chapman, S. C., \& Windhorst, R. A. 2003b, ApJ, 596, 5L

Conselice, C. J., et al. 2004, ApJ, 600, 139L

Conselice, C. J., Blackburne, J., \& Papovich, C. 2005a, ApJ, 620, 564

Conselice, C. J., Bundy, K., Ellis, R., Brichmann, J., Vogt, N., \& Phillips, A. 2005b, ApJ, 628, 160

Conselice, C. J. 2006a, MNRAS, 373, 1389

Conselice, C. J. 2006b, ApJ, 638, 686

Conselice, C. J., et al. 2007a, ApJ, 660, 55L

Conselice, C. J., et al. 2007b, arXiv:0708.1040

Daddi, E., et al. 2004, ApJ, 600, 127L

Drory, N. \& Fisher, D. B. 2007, ApJ, 664, 640

Ellis, R. S., Abraham, R., \& Dickinson, M. 2001, ApJ, 551, 111

Elmegreen, D. M., Elmegreen, B. G. \& Hirst, A. C. 2004, ApJ, 604, 21L

Elmegreen, D. M., Elmegreen, B. G., Rubin, D. S., \& Schaffer, M. A. 2005, ApJ, 631, 85

Elmegreen, D. M., Elmegreen, B. G., Ravindranath, S., \& Coe, D. A. 2007, ApJ, 658, 763

Elmegreen, B. G. \& Elmegreen, D. M. 2005, ApJ, 627, 632

Erb, D. K., et al. 2004, ApJ, 612, 122

Fontana, A., et al. 2004, A\&A, 424, 23

Forster Schreiber, N. M., et al. 2006, ApJ, 645, 1062

Genzel, R., et al. 2006, Nature, 442, 786

Giavalisco, M., et al. 2004, ApJ, 600, 93L

Immeli, A., et al. 2004, ApJ, 611, 20

Jogee, S., et al. 2004, ApJ, 615, 105L

Koo, D. C., et al. 2005, ApJS, 157, 175

Kormendy, J. \& Kennicutt, R. C., Jr. 2004, ARA\&A, 42, 603

Labbe, I., et al. 2003, ApJ, 591, 95L

Lanyon-Foster, M. M., Conselice, C. J., \& Merrifield, M. 2007, arXiv:0706.2622

Noguchi, M. 1999, ApJ, 514, 77

Papovich, C., et al. 2005, ApJ, 631, 101

Pope, A., et al. 2005, MNRAS, 358, 149

Ravindranath, S., et al. 2004, ApJ, 604, 9L

Ravindranath, S., et al. 2006, ApJ, 652, 963

Stanford, S. A., et al. 2004, AJ, 127, 131

Taylor-Mager, V., Conselice, C., Windhorst, R., \& Jansen, R. 2007, ApJ, 659, 162

Teplitz, H. I., et al. 2006, AJ, 132, 853

Windhorst, R., et al. 2002, ApJS, 143, 113

Vogt, N. P., et al. 1997, ApJ, 479, 121L

Wolf, C., et al. 2005, 630, 771 